\title{
Comparison of Path Loss near Homogeneous Medium, Layered Medium and Anatomically Correct Human Model
}

\author{
Laurens Roelens*, Wout Joseph, and Luc Martens \\ Department of Information Technology, Ghent University \\ Gaston Crommenlaan 8, box 201, B-9050 Gent, Belgium \\ http://www.wica.intec.ugent.be
}

\begin{abstract}
This paper presents a path loss model for two half-wavelength dipoles at $2.4 \mathrm{GHz}$ placed closely to a flat, homogeneous medium and compares the path loss near a flat homogeneous medium, a flat layered medium and an anatomically correct human body model.
\end{abstract}

\section{Introduction}

A Wireless Body Area Network (WBAN) is a network of which the nodes are situated in the clothes, on the body or under the skin of a person. These nodes are connected through a wireless communication channel and form a network that typically expands over the body of a person. According to the implementation, the nodes consist of sensors and actuators, placed in a star or multihop topology. A WBAN offers many promising, new applications in medicine, multimedia and sports, all of which make advantage of the unconstrained freedom of movement provided by a WBAN. The very complex shape and internal structure of the human body, however, make it impossible to derive a simple path loss (PL) model. Therefore, we start from a flat, homogeneous configuration of biological tissue from which we have extracted a PL model. In a second step, we analyse flat, layered media with anatomically correct thicknesses for each layer. Finally, a full whole-body model based on the Visible Human [1] is used and the PL is compared to the PL near homogeneous and layered media. All the simulations and measurements are performed at a frequency of $2.4 \mathrm{GHz}$, situated in the license-free Industrial, Scientific and Medical (ISM) frequency band. The PL in this paper is defined as $-\left|S_{21}\right|$, with $S_{21}$ being the scattering parameter representing the transmission loss.

\section{Flat Homogeneous Medium}

Using the EMSS FEKO simulator, based on the Method of Moments (MoM), we simulate horizontal half-wavelength dipoles placed above a flat, lossy and semiinfinite dielectric. Fig. 1 (a) shows the simulation setup. Tx and Rx represent the transmit and receive antenna, respectively. $d$ is the distance between the centres of the dipoles, $t$ is the thickness of the dipoles, $h$ is the height of the antenna centre above the medium and $\ell$ is the length of the dipoles. $\epsilon_{r}$ and $\sigma$ represent the relative permittivity and the conductivity $(\mathrm{S} / \mathrm{m})$ of the tissue-simulating medium, respectively. Identical half-wavelength dipoles are used at $2.4 \mathrm{GHz}$, with a length $\ell=0.46 \lambda=5.75 \mathrm{~cm}$ and a realistic diameter $t=1 \mathrm{~mm}$.

The simulations are compared with measurements performed with a vector network 
analyzer (Rohde \& Schwarz ZVR). A rectangular box phantom with dimensions $80 \times 50 \times 20 \mathrm{~cm}^{3}$, recommended by the CENELEC standard EN50383 [2], is filled with tissue simulating liquid. The transmit antenna $\mathrm{Tx}$ is fixed above the liquid surface, while the receive antenna $\mathrm{Rx}$ is moved above the liquid by means of a robot with a three-dimensional accuracy of $25 \mu \mathrm{m}$. The dipoles are parallel to each other and lined up for maximal power transfer.

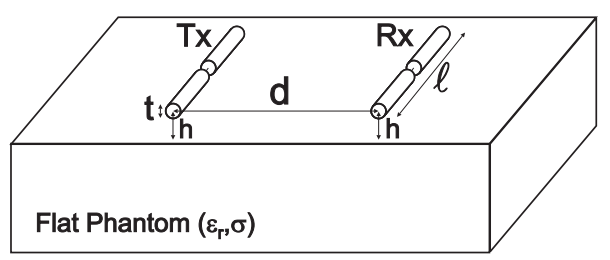

(a)

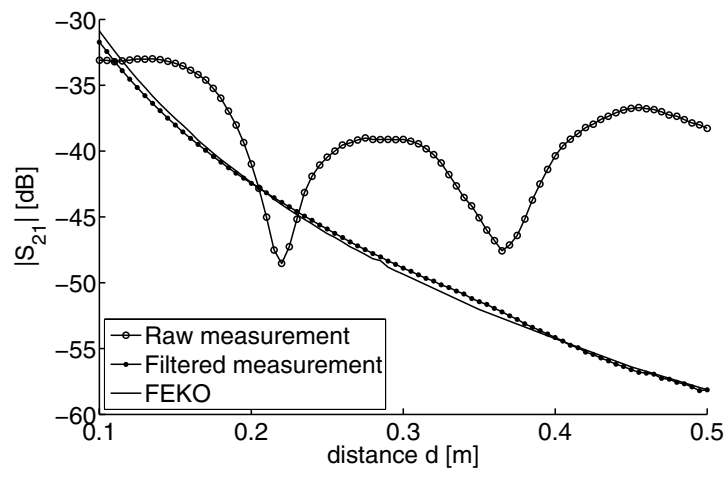

(b)

Figure 1: Measurement and simulation setup (a) and results for the homogeneous, flat medium (b).

Fig. 1 (b) shows measurement and simulation results for Tx and Rx both $1 \mathrm{~cm}$ above brain-simulating tissue $\left(\epsilon_{r}=34.3\right.$ and $\sigma=1.74 \mathrm{~S} / \mathrm{m} \mathrm{[3])}$ and distances up to $50 \mathrm{~cm}$. As the measurements are performed in a non-anechoic environment, resulting in undesired reflections, a de-embedding step is performed [4]. The raw measurement in Fig. 1 (b) shows the unprocessed results, which clearly suffer from reflections of the environment. After de-embedding, the filtered measurement is obtained. As can be seen, the filtered measurement shows excellent agreement with the simulated results in FEKO, quantified by a maximum deviation of only $0.6 \mathrm{~dB}$.

A series of simulations with the MoM tool for varying antenna heights $h$ from $5 \mathrm{~mm}$ up to $50 \mathrm{~mm}$ and distances up to $50 \mathrm{~cm}$ leads to the following semi-empirical $\mathrm{PL}$ model for brain tissue, based on the Friis free-space formula:

$$
\left.\frac{P_{r}}{P_{t}}(d, h)\right|_{d B}=\left|S_{21}\right|_{d B}=\left.P_{0}(h)\right|_{d B}-\left.n(h) d\right|_{d B}
$$

with

$$
\begin{array}{rlr} 
& n(h)=-25.0 h+4.0 \\
\left.P(h)\right|_{d B} & =7.7 \ln (h)-11.9, \quad h \leq 0.15 \lambda \\
& =388.7 h-49.4, \quad h>0.15 \lambda
\end{array}
$$

for brain tissue. $P_{r}$ and $P_{t}$ represent the received and transmitted power, respectively. $P_{0}$ is the PL at a reference distance $d_{0}$ (here: $d_{0}=40 \mathrm{~cm}$ ) and $n$ is the so called path loss exponent. This model was obtained through fitting of the simulation results. Similar models can be formulated for other biological tissues. 


\section{Flat Layered Medium}

A series of simulations was performed with different semi-infinite biological tissue configurations and compared with a layered combination of these tissues. The layered medium, consisting of skin, fat, muscle tissue and a layer representing an average of all the intestines, is shown in Fig. 2 (a) together with their dielectric properties [3]. The thicknesses for the layers of the back and leg models are chosen according to the anatomically correct model discussed in the next section. Fig. 2 (b) shows the simulation results for homogeneous and layered biological tissues as a function of the distance $d$ between the antennas $(h=5 \mathrm{~mm})$. The result for average intestines is not shown here because it almost coincides with the curve for muscle tissue. The curves for fat and muscle tissue are only shifted $1.4 \mathrm{~dB}$ on average, although their differences in $\epsilon_{r}$ and $\sigma$ are quite significant. According to [5], $\epsilon_{r}$ for the body ranges from 5.2 to 68.4 and $\sigma$ from $0.09 \mathrm{~S} / \mathrm{m}$ to $3.41 \mathrm{~S} / \mathrm{m}$ at $2.4 \mathrm{GHz}$. Hence, a large part of the human tissues will generate curves situated closely to the ones depicted in Fig. 2 for skin, fat and muscle tissue.

From Fig. 2, it is clear that the introduction of layers causes $\left|S_{21}\right|$ to deviate significantly from the homogeneous configurations. For the layered back configuration, $\left|S_{21}\right|$ is shifted up $2.1 \mathrm{~dB}$ on average, while for the layered leg configuration, $\left|S_{21}\right|$ is shifted down $6.1 \mathrm{~dB}$ on average compared to homogeneous fat tissue. Depending on the layer thicknesses, a homogeneous medium will thus over- or underestimate the PL compared to a layered medium.

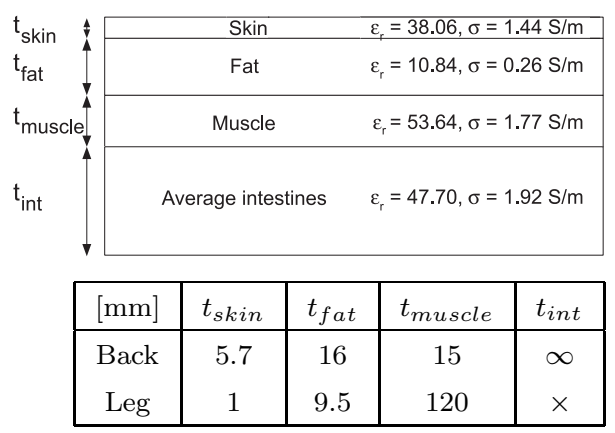

(a)

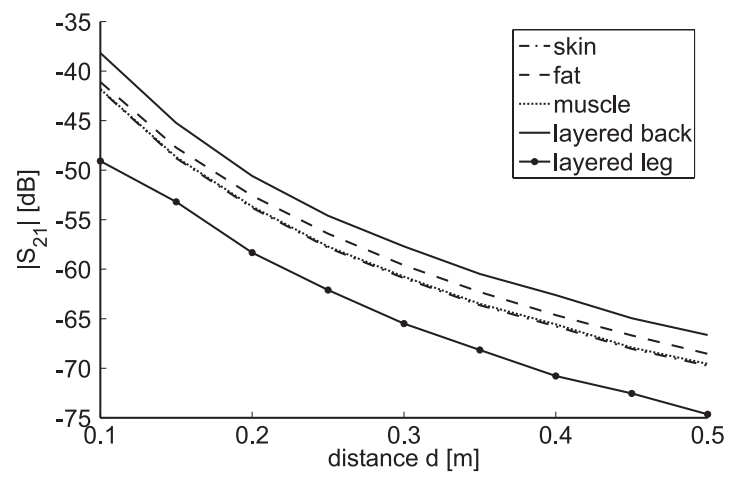

(b)

Figure 2: Configuration approximating the structure of a human back and leg (a) and comparison between homogeneous and layered biological tissue $(h=5 \mathrm{~mm})(\mathrm{b})$.

\section{Whole Body Configuration}

The results from the previous sections are now compared to the case of an anatomically correct configuration, using the 'Visible Human' model developed at Brooks Air Force Base Laboratories [1]. The model, shown in Fig. 3 (a), is simulated with the FDTD simulator SEMCAD. Tx and Rx are placed at a distance $h=5 \mathrm{~mm}$ from the back. Tx is fixed at the position shown in Fig. 3, while Rx is consecutively placed at the other indicated positions, separated $5 \mathrm{~cm}$ from each other. The results are shown in Fig. 3 (b). We see excellent agreement with the layered back case from 
the previous section for distances up to $35 \mathrm{~cm}$. The deviation for larger distances is due to the fact that the back is not perfectly flat over these distances. The flat, layered model thus provides a good estimation of the PL at flat regions of a human body.

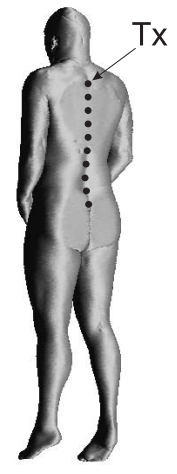

(a)

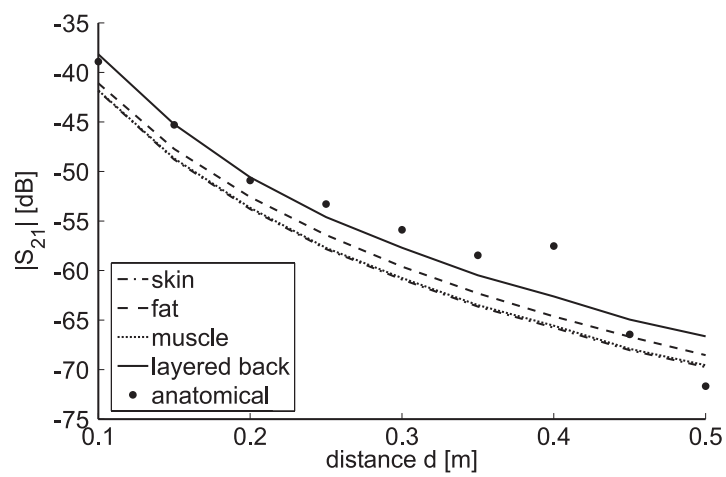

(b)

Figure 3: The 'Visible Human' with antenna positions (a) and comparison between flat homogeneous, flat layered and anatomically correct model $(h=5 \mathrm{~mm})(\mathrm{b})$.

\section{Conclusions}

The path loss near flat, homogeneous tissue at $2.4 \mathrm{GHz}$ was characterized and lead to a path loss model that shows excellent agreement with measurements and simulations. It was found that a homogeneous medium will over- or underestimate the path loss compared to a layered medium, depending on the thickness of the different layers. Simulations on an anatomically correct human body model showed that a flat layered medium provides a good estimate of the path loss at flat regions of the human body.

\section{References}

[1] J. M. Ziriax, D. Le Blanc, P. A. Mason, and W. D. Hurt, "Finite-difference timedomain for personal computers," in 21st Ann. Meeting of the Bioelectromagnetics Soc., Long Beach CA, June 1999, p. 57.

[2] CENELEC EN50838, "Basic Standard for the Calculation and Measurement of Electromagnetic Field Strength and SAR related to Human Exposure from Radio Base Stations and Fixed Terminal Stations for Wireless Telecommunications Systems $(110 \mathrm{MHz}-40 \mathrm{GHz}), 2002$.

[3] C. Gabriel, "Compilation of the dielectric properties of body tissues at RF and microwave frequencies," Brooks Air Force Base, San Antonio, Tech. Rep. AL/OE-RE-1996-0037, 1996, [Online] Available: http://www.fcc.gov/cgibin/dielec.sh.

[4] L. M. W. Joseph, L. Verloock, "Accurate low-cost measurement technique for occupational exposure assessment of base station antennas," IEE Electronics Letters, vol. 39, no. 12, pp. 886 - 887, June 2003. 


\section{IEEE Antennas and Propagation Society International Symposium 2006}
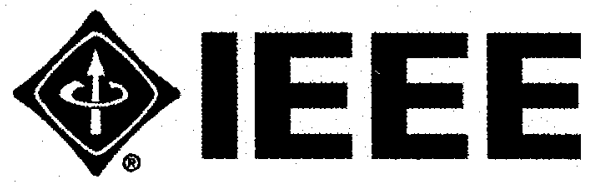

Copyright and Reprint Permissions: Abstracting is permitted with credit to the source. Libraries are permitted to photocopy beyond the limit of U.S. copyright law for private use of patrons those articles in this volume that carry a code at the bottom of the first page, provided the per-copy fee indicated in thecode is paid through Copyright Clearance Center, 222 Rosewood Drive, Danvers, MA 01923.

For other copying, reprint or republications permission, write to IEEE Copyrights Manager, IEEE Operations Center, 445 Hoes Lane, Piscataway, New Jersey USA 08854. All rights reserved.

IEEE Catalog Number:

ISBN:

Library of Congress No.:
$06 \mathrm{CH} 37758 \mathrm{C}$

1-4244-0123-2

$90-640397$ 


\title{
2006 IEEE Antennas and Propagation
} Society International Symposium

\author{
with

\section{USNC/URSI National Radio Science and AMEREM Meetings}

\section{Symposium Digest Volume 1 of 5}

\author{
9-14 July 2006
} Albuquerque Convention Center and Hyatt Regency Hotel Albuquerque, NM USA

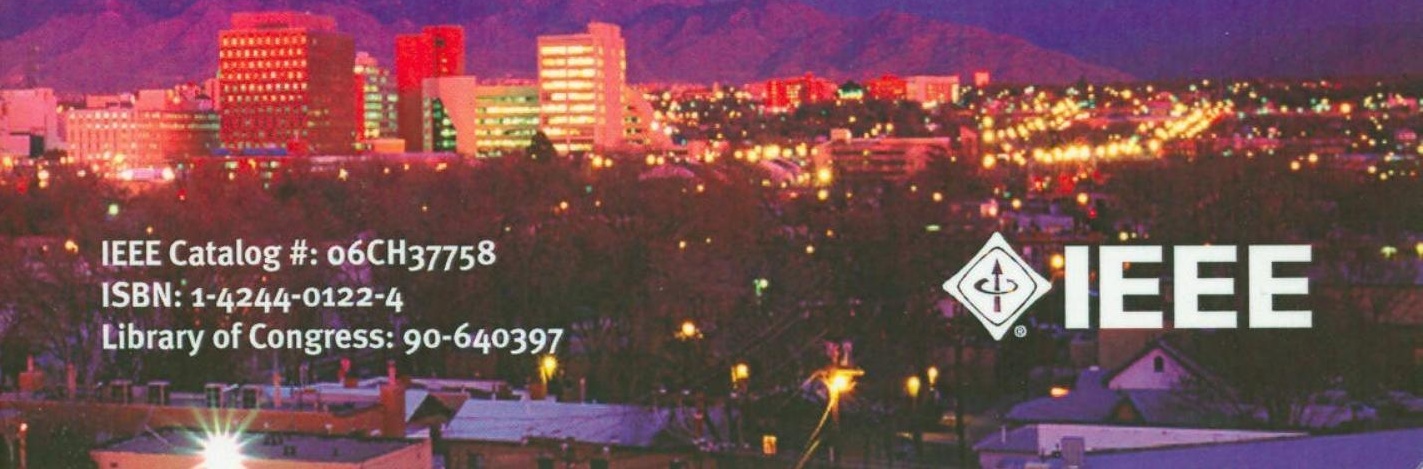

